\title{
New Application of Recognizing Thermal Images by Human Visual Perception
}

\author{
by Bo-Wen Wu* and Yi-Chin Fang**
}

\begin{abstract}
*Department Optometry, College of Medical Technology, Nursing and Wellbeing, Yuanpei University, wubwen@mail.ypu.edu.tw

**National Kaohsiung First University of Science and Institute of Electro-Optical Engineering,

yfang@nkfust.edu.tw
\end{abstract}

\begin{abstract}
Advances in optical and electronic technology can immensely reduce noise in images and greatly enhance human visual recognition. However, it is still difficult for human eyes to identify low resolution thermal images due to the limits imposed by psychological and physiological factors. In addition, changes in monitor brightness and lens resolution may also interfere with visual recognition abilities. To overcome the aforementioned limitations, we devised a suitable and effective recognition method which may help the military in revising the shape parameters of long-range targets. Modulation transfer function (MTF) was used as a basis to extend the visual characteristics of the human visual model and a new model was produced through incorporation with new shape parameters. The new model was then tested in experiments and the results showed that the accuracy is closer to the human eye.
\end{abstract}

\section{Human vision model}

In an earlier model, it was assumed that an entering brightness signal is first filtered by the optical matrix of the eye and then by the MTF of a lateral inhibition process. It is further assumed that the optical MTF is mainly determined by the eye lens and the discrete structure of the retina and that the MTF of the lateral inhibition is determined by neural processing. Figure 1 shows a block diagram of this model. For the sake of completeness, the external noise is also shown in this figure. External noises may, for instance, be from display noise present in a television image, or grain noise present in a photographic image [11-12]

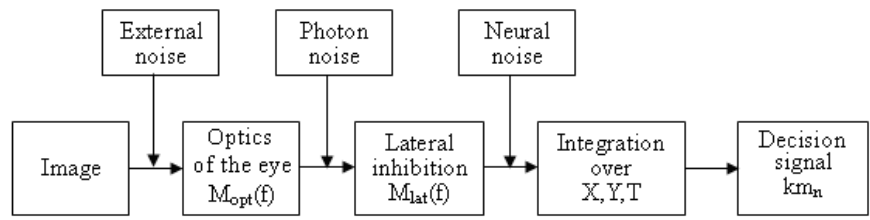

Figure 1 Block diagram of the processing of information and noise according to the human

After inserting binocular viewing factor $(\sqrt{2})$, internal noise and integration characteristics, the MTFeye for the human vision model are obtained[11], as in equation (1).

$$
\operatorname{MTF}_{\text {eye }}(f)=\frac{1}{m_{t}(f)}=\frac{M_{\text {opt }}(f) / k}{\sqrt{\frac{2}{T}\left(\frac{1}{X_{0}^{2}}+\frac{1}{X_{\max }^{2}}+\frac{f^{2}}{N_{\max }^{2}}\right)\left(\frac{1}{\eta p E}+\frac{\Phi_{0}}{1-e^{-\left(f / f_{0}\right)^{2}}}\right)}}
$$

The parameters in the model have the following typical values:

\section{Experiment design of shaping parameters related to human visual perception}

The current methods for predicting the ability of human observers to correctly interpret images of objects using a particular thermal imager depend on the thermal properties of the object and its background and rely, either directly or indirectly, on the measured minimum resolvable temperature difference (MRTD) [13]. Thus, Equation (1) does not take into account the shape parameter of the image when determining human visual perception. Because the human eye has poor sensitivity to high frequencies, thermal images in lack of high frequency are employed in the experiments to estimate the values needed for shape parameter correction.

Six simple shapes [13] (shapes which were found to be most frequently indistinguishable) of uniform intensity and equal area were shown to 100 observers. Each shape comprised 10000 pixels in an array of $200 \times 200$ pixels. The observers were seated approximately $25 \mathrm{~cm}$ from the monitor screen (Asus 20 inch. monitor) and instructed to identify the shapes. No fixed time was allotted for identification. The observers had to respond to all images and were allowed to make a guess when they could not identify the shape. All the images were viewed three times, and a record was made of each presentation, the observer's response, and the time taken for identification.

The monitor screen had a mean brightness of $34 \mathrm{~cd} / \mathrm{m}^{2}$. The observers consisted of graduate school students, aged between 18 and 22. Thermal images of the various shapes (hexagon, square, triangle, rectangle $(2 \times 3)$, rectangle $(1 \times 2)$, 
rectangle $(1 \times 3))$ have been degraded by decreasing temperature $\left(\Delta \mathrm{T}=0.8 \sim 0.4{ }^{\circ} \mathrm{C}\right)$. A diagrammatical representation of the experiment is shown in figure 2. Some of the data are presented in Table 1.

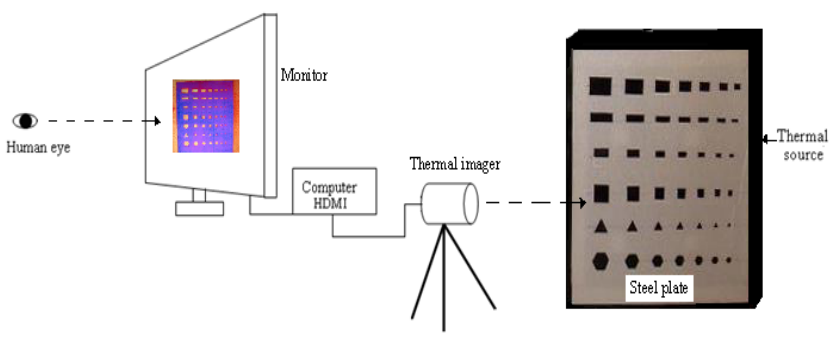

Figure 2 Diagrammatical representation of the shape parameter experiment

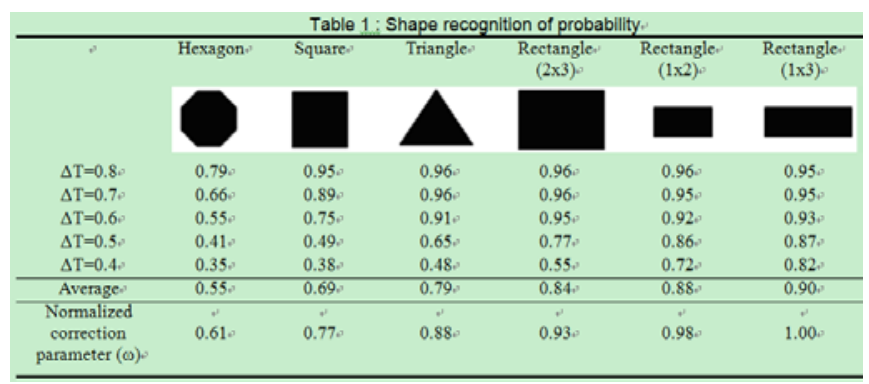

The true probability of shape recognition (observer performance) for each image was plotted against the temperature difference $(\Delta T)$ (Figure 3$)$. A unique curve was observed for each shape.

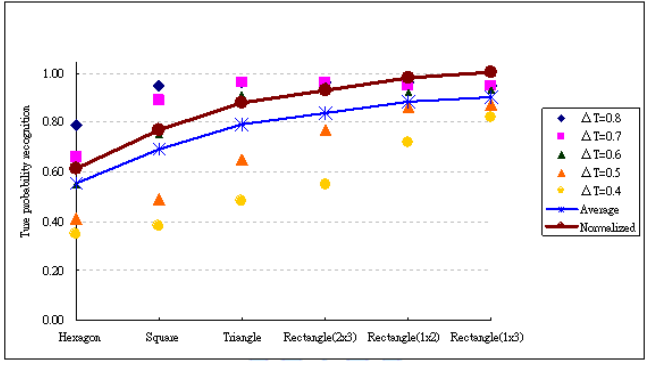

Figure 3 True probability of shape discrimination as a function of $\Delta T$ for different simple shapes degraded by decreasing temperature

The average image recognition rate is above $50 \%\left(\Delta \mathrm{T}=0.8 \sim 0.4{ }^{\circ} \mathrm{C}\right)$.

Normalized correction shape parameter (' $\omega$ ') is outlined in the Table 1 at bottom. The data curve fitting is obtained a equation (2).

$$
\omega=\left(1.29-1.18(\ln (x))^{-1}\right)^{2}
$$

The " $x$ " values are as follows: Hexagon $=10$, Square $=20$, Triangle $=30$, Rectangle $(2 \times 3)=40, \operatorname{Rectangle}(1 \times 2)=50$, Rectangle $(1 \times 3)=60$.

The following formula for the shape parameter correction of human vision model by binocular vision was obtained.

$$
\operatorname{MTF}_{\text {eye }}(f)=\frac{\left[\frac{M_{\text {opt }}(f)+\omega}{2}\right] / k}{\sqrt{\frac{2}{T}\left(\frac{1}{X_{0}^{2}}+\frac{1}{X_{\max }^{2}}+\frac{f^{2}}{N_{\max }^{2}}\right)\left(\frac{1}{\eta p E}+\frac{\Phi_{0}}{1-e^{-\left(f / f_{0}\right)^{2}}}\right)}} .
$$

\section{Conclusions}

Human recognition plays a vital role in military and remote sensing purposes. The properties of human vision recognition are comprehensively discussed in this research, including human MTF transmission and shape parameter correction. All relevant research and integral analysis of human eye recognition are statically studied with the help of MTF and shaping parameters.

The results of this study, both experimental and theoretical, theoretical human vision model were firstly defined and the need for a shape parameter correction (data curve fitting, equation 3) was stated in order to meet the demands of various applications that require accurate shape recognition. Analysis of the human eye MTF was studied in this research and then experimental results were statically analyzed. The theoretical and experimental results were in good agreement with each other. 